\title{
PROPER MOTIONS OF THE OUTER KNOTS OF THE HH 80/81/80N RADIO-JET
}

\author{
Josep M. Masqué ${ }^{1,2}$, Luis F. Rodríguez ${ }^{1}$, Anabella Araudo ${ }^{3}$, Robert Estalella ${ }^{4}$, Carlos Carrasco-González ${ }^{1}$, \\ Guillem Anglada ${ }^{5}$, Josep M. Girart ${ }^{6}$, and Mayra Osorio ${ }^{5}$ \\ ${ }^{1}$ Instituto de Radioastronomía y Astrofísica, Universidad Nacional Autónoma de México, Morelia 58089, México \\ ${ }^{2}$ Departamento de Astronomía, Universidad de Guanajuato, Apdo. Postal 144, 36000 Guanajuato, México \\ ${ }^{3}$ University of Oxford, Astrophysics, Keble Road, Oxford OX1 3RH, UK \\ ${ }^{4}$ Departament d'Astronomia i Meteorologia and Institut de Ciències del Cosmos (IEEC-UB), Universitat de Barcelona, \\ Martí i Franquès 1, E-08028 Barcelona, Catalunya, Spain \\ ${ }^{5}$ Instituto de Astrofísica de Andalucía (CSIC), Apartado 3004, E-18080 Granada, Spain \\ ${ }^{6}$ Institut de Ciències de l'Espai (CSIC-IEEC), Campus UAB, Carrer de Can Magrans, S/N, E-08193 Cerdanyola del Vallès, Catalunya, Spain \\ Received 2015 June 23; accepted 2015 October 6; published 2015 November 16
}

\begin{abstract}
The radio-knots of the Herbig-Haro $(\mathrm{HH}) 80 / 81 / 80 \mathrm{~N}$ jet extend from the $\mathrm{HH} 80$ object to the recently discovered Source 34 and has a total projected jet size of $10.3 \mathrm{pc}$, constituting the largest collimated radio-jet system known so far. It is powered by the bright infrared source IRAS 18162-2048 associated with a massive young stellar object. We report $6 \mathrm{~cm}$ JVLA observations that, compared with previous $6 \mathrm{~cm}$ VLA observations carried out in 1989 , allow us to derive proper motions of the $\mathrm{HH} \mathrm{80,} \mathrm{HH} 81$, and $\mathrm{HH} 80 \mathrm{~N}$ radio knots located about 2.5 pc away in projection from the powering source. For the first time, we measure proper motions of the optically obscured HH $80 \mathrm{~N}$ object providing evidence that this knot, along with $\mathrm{HH} 81$ and $\mathrm{HH} 80$ are associated with the same radio-jet. We also confirm the presence of Source 34, located further north of $\mathrm{HH} 80 \mathrm{~N}$, previously proposed to belong to the jet.We derived that the tangential velocity of $\mathrm{HH} 80 \mathrm{~N}$ is $260 \mathrm{~km} \mathrm{~s}^{-1}$ and has a direction in agreement with the expected direction of a ballistic precessing jet. The HH 80 and HH 81 objects have tangential velocities of 350 and $220 \mathrm{~km} \mathrm{~s}^{-1}$, respectively, but their directions are somewhat deviated from the expected jet path. The velocities of the $\mathrm{HH}$ objects studied in this work are significantly lower than those derived for the radio knots of the jet close to the powering source $\left(600-1400 \mathrm{~km} \mathrm{~s}^{-1}\right)$ suggesting that the jet is slowing down due to a strong interaction with the ambient medium. As a result, since $\mathrm{HH} 80$ and $\mathrm{HH} 81$ are located near the edge of the cloud, the inhomogeneous and low density medium may contribute to skew the direction of their determined proper motions. The HH 80 and $\mathrm{HH} 80 \mathrm{~N}$ emission at $6 \mathrm{~cm}$ is, at least in part, probably synchrotron radiation produced by relativistic electrons in a magnetic field of $1 \mathrm{mG}$. If these electrons are accelerated in a reverse adiabatic shock, we estimate a jet total density of $\lesssim 1000 \mathrm{~cm}^{-3}$. All of these features are consistent with a jet emanating from a high-mass protostar and make evident its capability of accelerating particles up to relativistic velocities.
\end{abstract}

Key words: Herbig-Haro objects - ISM: individual objects (IRAS 18162-2048) - ISM: jets and outflows

\section{INTRODUCTION}

The Herbig-Haro (HH) objects 80 and 81 (Reipurth \& Graham 1988) are among the brightest HH objects known. They lie at the southern edge of the dark molecular cloud L291 in Sagittarius, located at $1.7 \mathrm{kpc}$ of distance (Rodríguez et al. 1980), and are powered by the bright infrared source IRAS 18162-2048. Because of its high luminosity $\left(1.7 \times 10^{4} L_{\odot}\right)$, this source is believed to be associated with a massive protostar, or a cluster of protostars (Aspin \& Geballe 2007). The separation between $\mathrm{HH} \mathrm{80/81,} \mathrm{and}$ IRAS $18162-2048$ is $2.3 \mathrm{pc}$, the latter source having associated centimetric emission that appears as a thermal radio-jet pointing to $\mathrm{HH} 80$ and 81 (Martí et al. 1993). The central source is also associated with several molecular lines and compact millimeter continuum emission (Gómez et al. 2003; Fernández-López et al. 2011a, 2013) and has associated a disk with a radius of a few hundreds of AU (Fernández-López et al. 2011b; Carrasco-González et al. 2012). About 3 pc north of the central source there is $\mathrm{HH} 80 \mathrm{~N}$, a radio source that probably constitutes the northern counterpart of HH 80 (Martí et al. 1993). If $\mathrm{HH} 80 \mathrm{~N}$ is actually an $\mathrm{HH}$ object, its nondetection at optical wavelengths could be explained due to this object being deeply embedded in the molecular cloud. Further evidence of its $\mathrm{HH}$ nature is provided by the photochemical effects detected in a condensation of molecular gas and dust located ahead of HH 80N (Girart et al. 1994, 1998; Masqué et al. 2009). More recently, Masqué et al. (2012) reported a new radio source (Source 34) further north of $\mathrm{HH} 80 \mathrm{~N}$, closely coinciding with the direction of the jet. The fit of a precession jet model suggests that this source likely belongs to the $\mathrm{HH}$ complex (hereafter, the $\mathrm{HH} 80 / 81 / 80 \mathrm{~N}$ jet and, hence, that the jet extends beyond $\mathrm{HH} 80 \mathrm{~N}$. If confirmed, this would imply that the jet has a total projected length of $10.3 \mathrm{pc}$.

The characteristics of $\mathrm{HH} 80 / 81 / 80 \mathrm{~N}$ make this radio-jet a scaled up version of the jets emanating from low-mass protostars. Indeed, proper motion measurements yield projected velocities in the plane of the sky between 600 and $1400 \mathrm{~km} \mathrm{~s}^{-1}$ for the inner knots located a few arcsec away from the central source, and about $500 \mathrm{~km} \mathrm{~s}^{-1}$ for newly ejected condensations that traveled less than 1 arcsec in projection (Martí et al. 1995, 1998). Also, HH 80 and HH 81 proper motions were measured by Heathcote et al. (1998) using high spatial resolution optical observations that resolved the $\mathrm{HH}$ objects into several bright condensations. The measured proper motions presented a large dispersion of velocities (from 0 to $900 \mathrm{~km} \mathrm{~s}^{-1}$ ). In particular, proper motions of about $300 \mathrm{~km} \mathrm{~s}^{-1}$ were found for the brightest condensations in $\mathrm{HH} 80$ and HH 81.

The interaction of the jets with the ambient medium produces shocks that can accelerate electrons up to relativistic 
energies and subsequently radiate synchrotron emission (Araudo et al. 2007). This has been confirmed by the detection of linearly polarized radio emission at distances of $\sim 0.5 \mathrm{pc}$ from the central source of the HH $80 / 81 / 80 \mathrm{~N}$ jet (CarrascoGonzález et al. 2010). At larger distances, the sources HH 80, $\mathrm{HH}$ 81, and $\mathrm{HH} 80 \mathrm{~N}$ are also likely synchrotron emitters because of their negative spectral indices $\left(S_{\nu} \propto \nu^{-0.3}\right.$, being $S_{\nu}$ the flux at the frequency $\nu$ ), similarly to the radio-knots of jets found in Serpens (Rodríguez et al. 1989, 2005; Curiel et al. 1993), Cep A (Garay et al. 1996). and W3( $\left.\mathrm{H}_{2} \mathrm{O}\right)$ (Wilner et al. 1999). The presence of hot plasma and relativistic electrons downstream of the shock makes $\mathrm{HH}$ jets potential (thermal) $\mathrm{X}$ - and (non-thermal) $\gamma$-ray emitters (Raga et al. 2002; Bosch-Ramon et al. 2010; Araudo \& Rodríguez 2012). In this sense, Pravdo et al. (2004) detected thermal X-ray emission in $\mathrm{HH} 80$ and $\mathrm{HH} 81$ that was claimed to arise when the jet slams into the ambient material.

In this paper, we report proper motion measurements, made at radio wavelenghts for the first time, of the radio-knots $\mathrm{HH}$ $80, \mathrm{HH} 81$, and $\mathrm{HH} 80 \mathrm{~N}$, in order to confirm, first, that the three knots belong to the same jet and, second, that these $\mathrm{HH}$ objects have significantly slower proper motions than the radio knots located a few arcsec from the central source, indicating strong jet interactions with the ambient cloud. These results provide an important complement to previous proper motion studies of the $\mathrm{HH} 80 / 81 / 80 \mathrm{~N}$ jet near the center (Martí et al. 1995, 1998), as we investigate the jet behavior far away from the central source. Also, the results reported here allow us to estimate the magnitude of the magnetic field in equipartition with non-thermal particles $\left(B_{\mathrm{eq}}\right)$ and the jet density $\left(n_{\mathrm{j}}\right)$ at the position of the $\mathrm{HH}$ objects. We also report observations of the region northward of $\mathrm{HH} 80 \mathrm{~N}$ confirming the presence of Source 34 detected by Masqué et al. (2012).

\section{OBSERVATIONS AND RESULTS}

The new observations were carried out on 2013 September 6 using the JVLA interferometer in the hybrid $\mathrm{CnB}$ configuration. We observed in the $\mathrm{C}$ band $(6 \mathrm{~cm}$ wavelength, from 4.5 to $6.5 \mathrm{GHz}$ ) in continuum imaging mode with full polarization. We used the 8 bit sampler and set $3 \mathrm{~s}$ as the integration time. The flux and gain calibrators were 3C286 and J1820-2528, respectively. The bootstrapped $6 \mathrm{~cm}$ flux density of J1820 -2528 was $0.824 \pm 0.002 \mathrm{Jy}$ at the frequency of $5.36 \mathrm{GHz}$ (spectral index of $0.19 \pm 0.02$ ). We observed 3 fields, covering all of the jet length (i.e., from Source 34 to HH 80, see Figure 2 of Masqué et al. 2012). The northernmost field was centered on the highly luminous radio-source J1819-2036 (see Martí et al. 1993 and Masqué et al. 2012), while the two southern fields were chosen with the aim of reproducing the pointings performed in Martí et al. (1993; i.e., centered $\sim 2^{\prime}$ north and south, respectively, from the central source). The on-source time was three minutes per pointing with the exception of the J1819-2036 field, where only two minutes were employed.

The data were reduced using the Common Astronomy Software Applications (CASA) and the map was constructed with the CLEAN task with natural weighting. The resulting beamsize was 3 ! $9 \times 3$ !" 2 (P.A. = 44.5) and the achieved rms noise of the maps was $53 \mu \mathrm{Jy}_{\text {beam }}{ }^{-1}$ for the two southern fields. For the J1819-2036 field, the strong side-lobes of J1819 -2036 limited the dynamic range and increased the rms noise level to $\sim 500 \mu \mathrm{Jy}$ beam $^{-1}$. To solve this, we obtained a model of J1819-2036 from the calibrated data set and used it to self- calibrate the visibilities of the corresponding field. The J1819 -2036 source presents strong time variability (on a scale of a few hours) and a spectral index of approximately -0.5 . The integration time of the observations was shorter than the variability timescale of the source and the self-calibration was hardly affected by this. However, the J1819-2036 flux varied importantly in the $2 \mathrm{GHz}$ bandwidth. Thus, for the selfcalibration process, in the CLEAN task we employed three terms of the Taylor polynomial used to model the frequency structure across the bandwidth (nterms $=3$ ). The rms noise of the J1819-2036 field clearly improved reaching a value of $\sim 50 \mu \mathrm{Jy}_{\text {beam }^{-1}}$. The three fields were combined into a single mosaic using the linearmosaic function.

We also calibrated and imaged the $6 \mathrm{~cm}$ archival data of Martí et al. (1993), observed in September 1989 with the VLA in the $\mathrm{C}$ configuration. The details of the observations are given in that paper. We used CASA in a similar fashion as the observations presented above, but choosing uniform weighting for the final map. This gives a synthesized beam of 5 "! $8 \times 1$ "! 9 (P.A. $=-14^{\circ}$ ), more similar than the angular resolution of the 2013 observations, and an rms noise of $40 \mu \mathrm{Jy}$ beam $^{-1}$. This noise level is smaller than that of the Martí et al. (1993) $6 \mathrm{~cm}$ map and more appropriate for astrometric studies.

Figure 1 shows the $6 \mathrm{~cm}$ emission of the $\mathrm{HH} 80 / 81 / 80 \mathrm{~N}$ jet observed in 2013. Most of the sources found in Martí et al. (1993) are detected, including the ones belonging to the jet. The $6 \mathrm{~cm}$ map also shows emission at the position of Source 34 , with a signal-to-noise ratio of five previously reported by Masqué et al. (2012) and proposed to belong to the HH 80/81/ $80 \mathrm{~N}$ jet. This independent detection confirms the presence of this source. As seen in the top right panel of Figure 1, Source 34 appears splitted into two sources aligned roughly in the north-south direction.

In Table 1, we present the results of Gaussian fits to the main radio-knots of the $\mathrm{HH} 80 / 81 / 80 \mathrm{~N}$ jet (except for Source 34) from the $6 \mathrm{~cm}$ map obtained in 2013 convolved to the final beam size of $6 !^{\prime \prime} 0 \times 4 !^{\prime \prime} 0\left(\mathrm{PA}=-14^{\circ}\right)$, which is more appropriate for a comparison with the Martí et al. (1993) $6 \mathrm{~cm}$ map. Comparing the fluxes of this table with those of Martí et al. (1993), we assess a flux drop of $\sim 40 \%$ for HH 81, clearly above the flux calibration uncertainty of $10 \%$, even taking into account the different bandwidth of the data sets. The HH 80 object has an integrated flux roughly constant in the time baseline of 24 years. However, as seen in the lower right panel of Figure 1, the 2013 map displays HH 80 slightly more extended to the southwest, which is balanced by a decrease in the intensity peak with respect to the 1989 map. Both morphological and flux variations are common in the $\mathrm{HH}$ objects and are attributed to changes in the internal structure of the shock (e.g., HH 1-2 Rodríguez et al. 2000). On the other hand, the central source does not show significant variability as is typical for the central sources of thermal radiojets (e.g., Anglada 1996).

The proper motions of the radio-knots of the HH 80/81/80N jet were derived from the $6 \mathrm{~cm}$ maps of the 1989 and 2013 data convolved to the same final beam $\left(6\right.$ " $0 \times 4$ "! 0 ; P.A. $\left.=-14^{\circ}\right)$. Then, the fields corresponding to the northern and southern pointings of Martí et al. (1993; the J1819-2036 field has no 1989 observations) were aligned separately using a selected set of reference sources in the field detected in both epochs, not belonging to the jet (i.e., assumed to be stationary), and detected with an $\mathrm{S} / \mathrm{N}$ ratio greater than nine. This is a better 


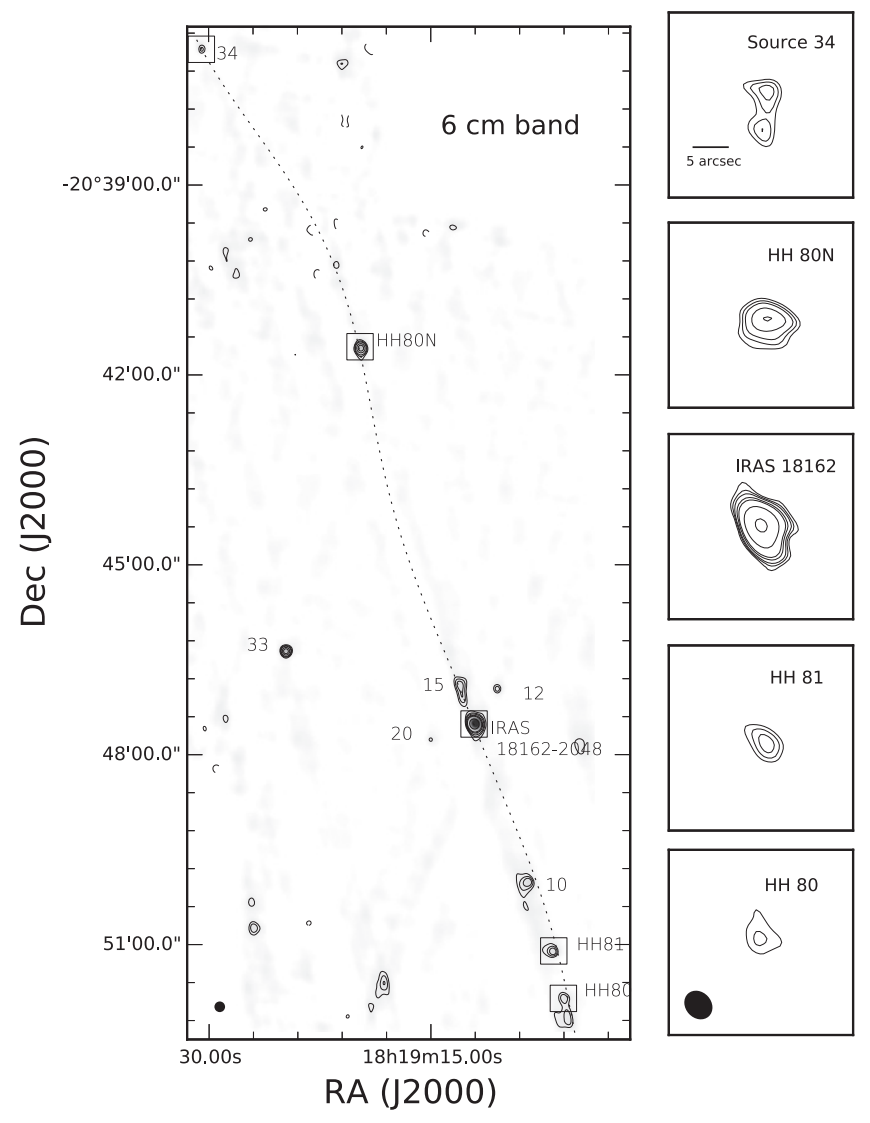

Figure 1. Left panel: map at $6 \mathrm{~cm}$ of the $\mathrm{HH} 80 / 81 / 80 \mathrm{~N}$ jet observed in 2013 with the JVLA in the CnB configuration convolved to a beamsize of $8^{\prime \prime}$. Contours are $-4,4,5,6,8,10,20,50$, and 100 times the rms noise level of the map $\left(19 \mu \mathrm{Jy}_{\text {beam }}{ }^{-1}\right)$. The radio knots are labeled following the nomenclature of Martí et al. (1993) and Masqué et al. (2012). The squares show the field of view displayed in the right panels $(20 \mathrm{arcsec})$. The dotted line represents the best-fit model of a precessing jet (model B of Masqué et al. 2012). Right panels: zoom of the $6 \mathrm{~cm}$ map obtained with natural weighting from the 2013 observations, which gives a synthesized beamsize of 3 !" $9 \times 3$ !" $2(\mathrm{PA}=44.5)$, of (from top to bottom) Source 34, HH 80N, IRAS 18162-2048, HH 81, and $\mathrm{HH} 80$. Contours are $-4,4,5,6,8,10,20,50$, and 100 times the rms noise of the map $\left(55 \mu \mathrm{Jy} \mathrm{beam}^{-1}\right)$. For Source 34 and $\mathrm{HH} 80$ contours -3 and 3 have been added. The scale of the maps is shown in the top panel.

choice than adopting the position of the central source as reference, which has periodic ejections of new condensations that can slightly modify its morphology affecting the fitting position of the centroid of this source. For the northern field, sources 12, 20, and 33 were used as reference while for the southern field, the same sources plus the sources 2 and 16 were used (these sources follow the nomenclature of Martí et al. 1993 and Masqué et al. 2012). These latter sources are located outside of the field of view of the left panel of Figure 1 and some arcminutes away from the pointing center. In order to prevent a bad fit position due to source smearing, we corrected the maps for the response of the primary beam. Sources 2 and 16 , despite falling close to the noisy edge of the primary beam, are bright enough to be detected with a good signal-to-noise ratio. The position of the sources was determined by Gaussians fits. Then, averaging the derived positions for the reference sources we determined a reference position for each epoch and pointing. Using this technique, we found shifts between the maps of the two epochs $(\Delta x, \Delta y)$ of $(-0.44,-0.24)$ and $(-0.36,-0 . .07)$ for the northern and southern fields, respectively. The rms of the difference in position $(\delta x, \delta y)$ of the reference sources between the 1989 and 2013 epochs, also known as the alignment error, were $(0$ !' 20, 0 !' 14) and (0!" 18 , 0 ". 27) for the northern and southern fields, respectively.

Once the reference positions of the 1989 and 2013 epochs were determined, the same Gaussian fitting as that above was employed to measure the relative positions of the radio-knots of the jet from the reference position of their respective field. Finally, we compared the relative positions of the radio-knots between the two epochs obtaining their displacement in 24 years. To derive the total error of the displacement of the sources, the uncertainties in the fit of the position determination of the two epochs, typically between 0 ! 1 and 0 .' 2 , were added quadratically to the alignment error. To obtain the total uncertainty in the source displacement along the PA of the motion, as well as the uncertainty of the PA itself, error propagation was applied. The two aligned images showing the observed angular displacement between the two epochs of observation (separated by 24 year) of the most important objects of the jet are presented in Figure 2. The derived motions are represented by arrows, with the ellipses corresponding to the error of the motion, whose values are presented in Table 1. From the measured displacement, we derive proper motion

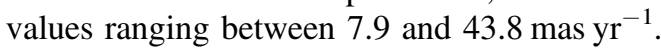

Our tangential velocities derived for the radio sources $\mathrm{HH} 80$ $\left(351 \pm 104 \mathrm{~km} \mathrm{~s}^{-1}\right), \mathrm{HH} 81\left(223 \pm 85 \mathrm{~km} \mathrm{~s}^{-1}\right)$, and $\mathrm{HH} 80 \mathrm{~N}$ $\left(263 \pm 71 \mathrm{~km} \mathrm{~s}^{-1}\right)$ have similar values to those of the brightest knots of the southern $\mathrm{HH}$ objects, $\mathrm{HH} 80 \mathrm{~A}\left(334 \pm 23 \mathrm{~km} \mathrm{~s}^{-1}\right)$ and $\mathrm{HH} 81 \mathrm{~A}\left(370 \pm 17 \mathrm{~km} \mathrm{~s}^{-1}\right)$ measured by Heathcote et al. (1998) from optical observations. As seen in Figure 2, the direction of the motion of $\mathrm{HH} 80 \mathrm{~N}$ is in good agreement with the expected direction for a ballistic precessing jet. The $\mathrm{HH} 80$ proper motion is somewhat deviated to the west with respect to the jet path but still traveling away from the central source. On the other hand, the direction of the $\mathrm{HH} 81$ proper motion is clearly not tangent to the jet path. The proper motions derived by Heathcote et al. (1998) for individual condensations in HH 80 and 81 also show considerable dispersion in velocity and direction. Finally, within the uncertainties, we find no significant displacement for the central source, as expected.

\section{DISCUSSION}

\subsection{The HH Nature of $\mathrm{HH} 80 \mathrm{~N}$}

$\mathrm{Up}$ to date, the likely $\mathrm{HH}$ nature of $\mathrm{HH} 80 \mathrm{~N}$ and its association with the jet emanating from IRAS 18162-2048 was based on its position aligned with the jet knots, with a very low probability for $\mathrm{HH} 80 \mathrm{~N}$ being a background source. In this work, we report for the first time proper motions of HH 80N. The proper motions point in a direction consistent with a ballistic motion of a jet element (see Figure 2). This provides evidence that $\mathrm{HH} 80 \mathrm{~N}, \mathrm{HH} 80$, and $\mathrm{HH} 81$ belong to the same jet, constituting the $\mathrm{HH} 80 / 81 / 80 \mathrm{~N}$ jet system. Furthermore, the similarity of the $\mathrm{HH} 80,81$, and $80 \mathrm{~N}$ spectral indices (approximately - 0.3, Martí et al. 1993; Masqué et al. 2012), their comparable tangential velocities (see Table 1) and approximate symmetric positions with respect to the central source suggest that their emission is generated under the same mechanism. Moreover, they were likely produced in the same major ejection event of the driving source. 
Table 1

Parameters at $6 \mathrm{~cm}$ of Selected Radio Knots

\begin{tabular}{|c|c|c|c|c|c|c|c|}
\hline \multirow[b]{2}{*}{ Source } & \multicolumn{2}{|c|}{ Peak Position $^{\mathrm{a}}$} & \multirow{2}{*}{$\begin{array}{c}S_{\nu}(6 \mathrm{~cm})^{\mathrm{a}} \\
\quad(\mathrm{mJy})\end{array}$} & \multicolumn{2}{|c|}{ Displacement $^{\mathrm{b}}$} & \multirow{2}{*}{$\begin{array}{c}\text { Velocity } \\
\left(\mathrm{km} \mathrm{s}^{-1}\right)\end{array}$} & \multirow{2}{*}{$\begin{array}{l}\text { P.A. }^{\mathbf{d}} \\
\text { (deg.) }\end{array}$} \\
\hline & $\alpha(\mathbf{J} 2000)$ & $\delta(\mathrm{J} 2000)$ & & $\Delta_{\alpha}\left({ }^{\prime \prime}\right)$ & $\Delta_{\delta}\left({ }^{\prime \prime}\right)$ & & \\
\hline HH 80 & $18^{\mathrm{h}} 19^{\mathrm{m}} 06^{\mathrm{s}} 04$ & $-20^{\circ} 51^{\prime} 51^{\prime \prime} 09$ & $1.3 \pm 0.1$ & $-0.75 \pm 0.30$ & $-0.74 \pm 0.32$ & $351 \pm 104$ & $-135 \pm 28$ \\
\hline HH 81 & $18^{\mathrm{h}} 19^{\mathrm{m}} 06^{\mathrm{s}} 69$ & $-20^{\circ} 51^{\prime} 06^{\prime \prime} 11$ & $1.1 \pm 0.1$ & $0.65 \pm 0.25$ & $-0.12 \pm 0.30$ & $223 \pm 85$ & $101 \pm 38$ \\
\hline IRAS 18162 & $18^{\mathrm{h}} 19^{\mathrm{m}} 12^{\mathrm{s}} 08$ & $-20^{\circ} 47^{\prime} 31^{\prime \prime} 00$ & $4.9 \pm 0.1$ & $0.07 \pm 0.20$ & $0.17 \pm 0.28$ & $63 \pm 89$ & $157 \pm 91$ \\
\hline $\mathrm{HH} 80 \mathrm{~N}$ & $18^{\mathrm{h}} 19^{\mathrm{m}} 19^{\mathrm{s}} 75$ & $-20^{\circ} 41^{\prime} 34^{\prime \prime} 85$ & $2.3 \pm 0.1$ & $0.13 \pm 0.30$ & $0.78 \pm 0.211$ & $263 \pm 71$ & $10 \pm 36$ \\
\hline
\end{tabular}

Notes.

a Derived from Gaussian fits to the sources of the $6 \mathrm{~cm}$ map obtained from the 2013 data convolved to 6 . $^{\prime \prime} \times 44^{\prime \prime} 0\left(\mathrm{PA}=-14^{\circ}\right)$ as the resulting beam.

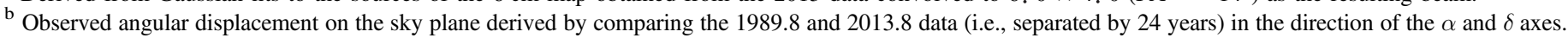

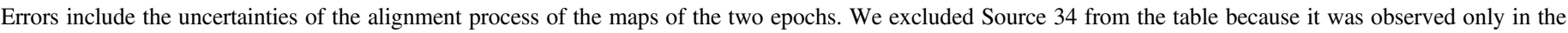
2013 epoch.

${ }^{\mathrm{c}}$ For an adopted distance of $1.7 \mathrm{kpc}$.

${ }^{\mathrm{d}}$ Position angle, measured from north to east.

\subsection{Synchrotron Emission From the HH Objects}

The emission of the inner knots studied by Martí et al. (1995, 1998), corresponding to recent ejections, is produced by internal shocks of the jet. Thus, their measured velocity is representative of the jet velocity. On the other hand, the emission of $\mathrm{HH} 80$ and 81 corresponding to older ejections arises from the interaction of the jet with the ambient cloud, owing to their known $\mathrm{HH}$ nature. As we mentioned in the previous section, $\mathrm{HH} 80 \mathrm{~N}$ likely has the same nature as $\mathrm{HH} 80$ and 81 and, hence, its emission is expected to be produced also by the jet interaction with the medium. The qualitative difference between the measured tangential velocities of the outer radio-knots $\mathrm{HH} 80, \mathrm{HH} 81$, and $\mathrm{HH} \quad 80 \mathrm{~N}$ $\left(\sim 223-351 \mathrm{~km} \mathrm{~s}^{-1}\right)$ and the inner radio knots ( 600-1400 $\mathrm{km} \mathrm{s}^{-1}$, Martí et al. 1995) implies that the ambient material, at least in some parts of the cloud, contributes significantly to slowing down the jet material. This is supported by the high bolometric luminosity of the outer $\mathrm{HH}$ objects, which suggests that strong interactions of a high velocity flow with stationary obstacles in the cloud. Certainly, the fading luminosity of $\mathrm{HH} 81$ and the change in morphology of $\mathrm{HH} 80$, as it escapes from the cloud and possibly expands into a low pressure medium, makes evident the important role of the ambient cloud in the emission of these $\mathrm{HH}$ objects.

The interaction of the jet with the ambient medium produces two shocks: a bow shock in the molecular cloud and a Mach disk (or reverse shock) in the jet. The shocked matter from the molecular cloud and jet are separated by an (unstable) contact discontinuity. This whole system propagates in the ambient medium at the bow shock speed $v_{\text {bs }}$ (Blondin et al. 1989) that is assumed to be the derived velocities for the HH objects shown in Table 3. Radiative shocks are those in which the plasma behind the shock emits thermal radiation in a distance (i.e., also called the cooling distance, $d_{\text {cool }}$ ) shorter to the jet radius, for which the source emitting size $l$ is usually adopted. In the opposite case, $d_{\text {cool }}>l$, the shock is non-radiative (i.e., adiabatic).

Heathcote et al. (1998) pointed out that significant parts of HH 80 and 81 must be adiabatic. Also, these authors concluded that there are some caveats with the assumption of $\mathrm{HH} 80$ and 81 as conventional bow shocks, specially the latter. Beyond HH 80, the jet escapes from the cloud and expands to a low density medium, resulting in a large dispersion of the measured proper motions for the fainter condensations (see Figure 8 of
Heathcote et al. 1998). The derived velocity of HH 80 suggests that radio observations basically include emission from $\mathrm{HH}$ $80 \mathrm{~A}$, even though the emitting region of the $\mathrm{HH}$ object part escaping from the cloud could contribute to skewing our proper motion determination to the southwest (i.e., the direction where the jet reaches the edge of the cloud). This effect probably tends to be more prominent with time. Similarly, in HH 80, there is a fainter condensation (HH 81B) located eastward from the brightest one (HH 81A) and radio observations possibly pick up the former. A possible explanation for the observed motion is a change in brightness of $\mathrm{HH} 81 \mathrm{~A}$ relative to $\mathrm{HH} 81 \mathrm{~B}$ during the time baseline inspected in this study. This would shift the measured centroid of the emission to the east resulting in the appearance that the bulk motion is moving toward this direction. On the other hand, the local conditions of $\mathrm{HH} 80 \mathrm{~N}$, deeply embedded in the cloud with constant confining pressure, are different than those of $\mathrm{HH} 80$ and 81 . Below, we discuss the different possible scenarios to explain the jet interaction with the cloud and the produced emission at $\mathrm{HH} 80 \mathrm{~N}$. In particular, we will consider that the synchrotron emission at $6 \mathrm{~cm}$ is produced by relativistic electrons with energies $\sim 60 m_{e} c^{2}(B / \mathrm{mG})^{-0.5}$ in a magnetic field $B$.

In Figure 3, we show the dependence between jet parameters estimated at the $\mathrm{HH} 80 \mathrm{~N}$ position. Considering the density of the molecular cloud, $n_{\mathrm{mc}, 80 \mathrm{~N}} \simeq 5 \times 10^{3} \mathrm{~cm}^{-3}$ (J. M. Masqué et al. 2015, in preparation), and its bow shock velocity $v_{80 \mathrm{~N}}=263 \mathrm{~km} \mathrm{~s}^{-1}$, the ambient shocked material emits thermal emission over a distance of $d_{\text {cool,bs }} \sim 22 \mathrm{AU}$ behind the shock (Raga et al. 2002). Given that $d_{\text {cool }}$ in the bow shock is much smaller than the emitting size $l \sim 3000 \mathrm{AU}$ (red-dashed line in Figure 3), the bow shock must be radiative. In such a case, the synchrotron emission at $6 \mathrm{~cm}$ could be produced by the compression of cosmic rays in the molecular cloud, as in the case of supernova remnants in the radiative stage (Chevalier 1999), or by particles accelerated via second-order Fermi acceleration (Ostrowski 1999).

The HH $80 \mathrm{~N}$ synchrotron emission can also be produced in the jet in the reverse shock region (Mach disc). Considering the jet velocity $v_{\mathrm{j}}$ as a free parameter, we calculate the total jet density $n_{\mathrm{j}} \sim n_{\mathrm{mc}, 80 \mathrm{~N}} /\left(v_{\mathrm{j}} / v_{80 \mathrm{~N}}-1\right)^{2}$ (green solid line in Figure 3) and the reverse shock velocity for $\mathrm{HH} 80 \mathrm{~N}$ as $v_{\mathrm{rs}, 80 \mathrm{~N}} \sim v_{\mathrm{j}}$ - $(3 / 4) v_{80 \mathrm{~N}}$. Therefore, comparing the thermal cooling length downstream the Mach disk $\left(d_{\text {cool,rs }}\right)$ with $l$, we can see in Figure 3 (green dashed line) that the Mach disk is non-radiative 

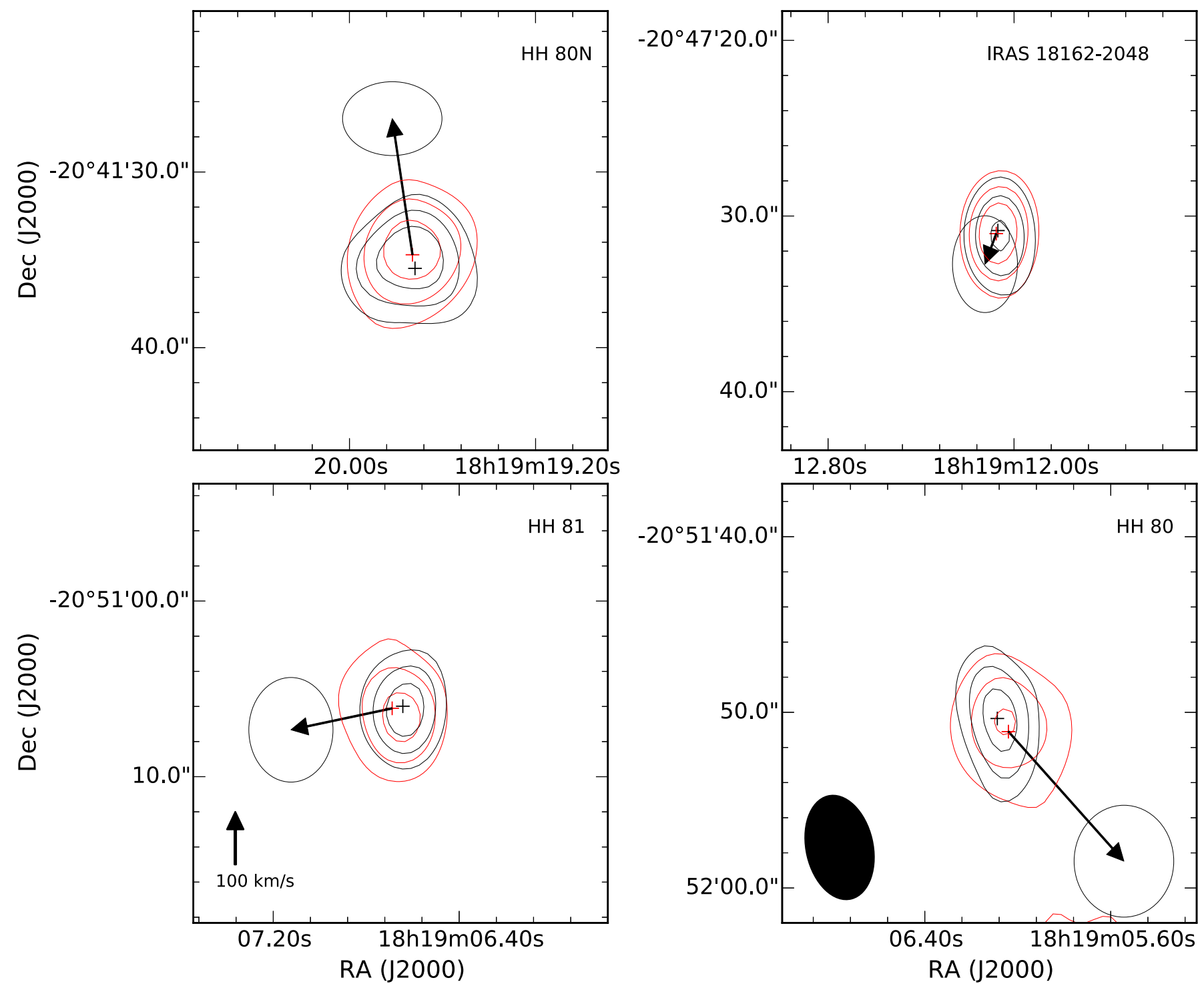

Figure $2.6 \mathrm{~cm}$ emission corresponding to the 1989 observations (black contours) and 2013 observations (red contours) of HH $80 \mathrm{~N}$ (top left panel), IRAS 18162 - 2048 (top right panel), HH 81 (bottom left panel), and HH 80 (bottom right panel). The maps have been aligned with the offsets given in Section 2 and convolved to the same final beam $\left(6^{\prime \prime} 0 \times 4{ }^{\prime \prime} 0 ; \mathrm{PA}=-14^{\circ}\right)$ that is shown in the bottom left corner of the bottom right panel. The contours are $50 \%$, $70 \%$, and $90 \%$ times the representative peak value of the object (HH 80N: 0.8 mJy beam $^{-1}$; IRAS 18162-2048: 3 mJy beam $^{-1} ; \mathrm{HH} 81: 1.0$ (1989) and 0.5 (2013) mJy beam ${ }^{-1}$; HH 80: 0.50 (1989) and $\left.0.39(2013) \mathrm{mJy}_{\text {beam }}{ }^{-1}\right)$. The arrows represent the magnitude and direction of the derived proper motions and the ellipses represent the uncertainties.

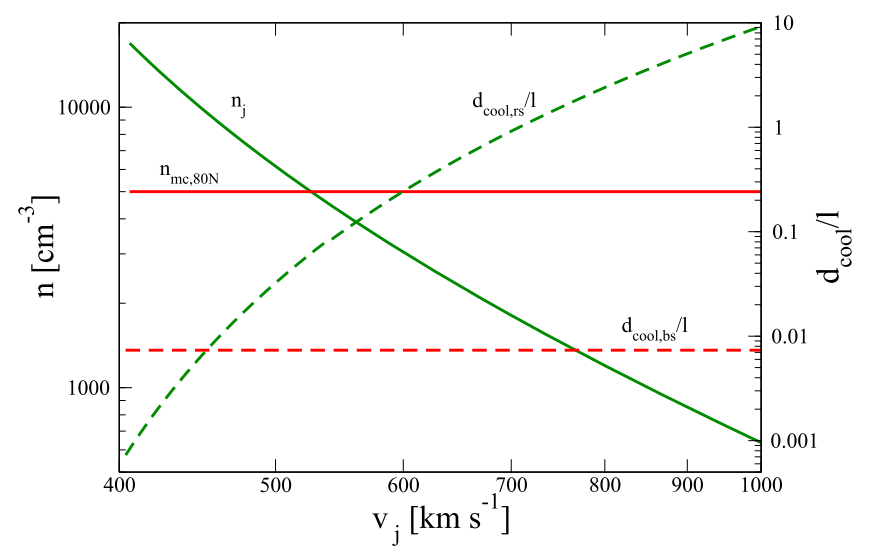

Figure 3. Left axis: jet (green, solid line) and molecular cloud densities (red solid line). Right axis: reverse- (green dashed line) and bow-shock thermal cooling length (red dashed line) in units of the source emitting size. The bow shock is radiative $\left(d_{\text {cool,bs }} / l<1\right)$ for all possible values of $v_{\mathrm{j}}$ while the reverse shock is adiabatic $\left(d_{\text {cool,rs }} / l>1\right)$ when $v_{\mathrm{j}} \gtrsim 800 \mathrm{~km} \mathrm{~s}^{-1}$. $\left(d_{\text {cool,rs }}>l\right)$ when $v_{\mathrm{j}} \gtrsim 800 \mathrm{~km} \mathrm{~s}^{-1}$. These values fall within the range of values found by Martí et al. (1995; 600-1400 $\mathrm{km} \mathrm{s}^{-1}$ ) that are possibly representative of the jet velocity. As seen in the figure, this implies jet densities of $\lesssim 1000 \mathrm{~cm}^{-3}$ (but probably greater than $500 \mathrm{~cm}^{-3}$ ). The inferred large jet velocities and densities are those expected for a jet emanating from a high mass protostar. Thus, the scenario of having synchrotron emission in the reverse shock of the jet is the most likely. In this case, relativistic electrons are accelerated via the Fermi I acceleration mechanism (Bell 1978) and emit synchrotron emission with an spectral index $\leqslant-0.5$, being the observed flat spectrum in $\mathrm{HH} 80 \mathrm{~N}(\alpha \sim-0.3)$ the result of thermal contamination. Assuming that relativistic electrons follow a power-law energy distribution $\propto E^{-2}$ that terminates at $E_{\min }=10 m_{e} c^{2}$, the magnetic field in equipartition with nonthermal electrons and protons is $B_{\mathrm{eq}} \sim 1 \mathrm{mG}$. If we adopt the energy density of non-thermal particles $U_{\mathrm{nt}}$ as $B^{2} /(8 \pi)$, we found that only a small fraction $(\sim 1 \%)$ of the jet kinetic energy 
density $U_{\text {kin }} \sim 5 \times 10^{-6}\left(n_{\mathrm{j}} / 500 \mathrm{~cm}^{-3}\right)\left(v_{\mathrm{j}} / 1000 \mathrm{~km} \mathrm{~s}^{-1}\right)^{2} \mathrm{erg}$ $\mathrm{cm}^{-3}$, is converted into non-thermal energy in the shock downstream region (e.g., Park et al. 2015).

\section{SUMMARY}

We carried out $6 \mathrm{~cm}$ observations of the $\mathrm{HH} 80 / 81 / 80 \mathrm{~N}$ radiojet with the JVLA at $\mathrm{C}$ configuration. These observations, combined with previous VLA observations obtained with the same observing band and configuration as ours, allow us to derive proper motions for the $\mathrm{HH} 80, \mathrm{HH} 81$, and $\mathrm{HH} 80 \mathrm{~N}$ radio-knots of the jet. The measured proper motion of $\mathrm{HH} 80 \mathrm{~N}$ suggests that it belongs to the $\mathrm{HH} 80 / 81 / 80 \mathrm{~N}$ radio-jet constituting the northern counterpart of the $\mathrm{HH} 80$ and 81 objects. We also confirm the presence of Source 34 previously reported by Masqué et al. (2012). This supports the possibility that the jet extends further north of $\mathrm{HH} 80 \mathrm{~N}$. The derived velocities for $\mathrm{HH} 80$ $\left(351 \mathrm{~km} \mathrm{~s}^{-1}\right)$, HH $81\left(223 \mathrm{~km} \mathrm{~s}^{-1}\right)$, and $\mathrm{HH} 80 \mathrm{~N}\left(263 \mathrm{~km} \mathrm{~s}^{-1}\right)$ are quantitatively slower than those derived by Martí et al. (1995) for the inner knots of the jet (600-1400 $\mathrm{km} \mathrm{s}^{-1}$ ) corresponding to very recent ejections. This implies that the cloud medium contributes significantly in slowing down the jet material at the position of these $\mathrm{HH}$ objects. Indeed, the measured direction of the proper motions of $\mathrm{HH} 80$ and $\mathrm{HH} 81$ makes evident the importance of the medium in the emission of these $\mathrm{HH}$ objects: they are located in the edge of the cloud and possibly expanding to a low pressure medium. As a consequence, they suffer morphological changes that could alter our proper motion determination. On the other hand, $\mathrm{HH} 80 \mathrm{~N}$ is deeply embedded in the cloud with a more constant confining pressure. Estimating the nature of the shock of $\mathrm{HH} 80 \mathrm{~N}$, we found that it must be adiabatic and its emission is partly synchrotron radiation produced by electrons accelerated up to relativistic velocities in the reverse shock in the jet. We argue that the jet velocities must be $\gtrsim 800 \mathrm{~km} \mathrm{~s}^{-1}$, the derived jet density is $\lesssim 1000 \mathrm{~cm}^{-3}$, and the magnetic field in the shocks is $\sim 1 \mathrm{mG}$. The work presented here provides further evidence for particle acceleration in $\mathrm{HH} \mathrm{80,81,}$ and $80 \mathrm{~N}$ that produces the observed non-thermal emission.

The JVLA observations presented here were part of NRAO program 13B-386. The National Radio Astronomy Observatory is a facility of the National Science Foundation operated under cooperative agreement by Associated Universities, Inc.

\section{REFERENCES}

Anglada, G. 1996, in ASP Conf. Ser. 93, Radio Emission from the Stars and the Sun, ed. A. R. Taylor \& J. M. Paredes (San Francisco, CA: ASP), 3

Araudo, A. T., \& Rodríguez, L. F. 2012, in AIP Conf. Ser. 1505, High Energy Gamma-Ray Astronomy, ed. F. A. Aharonian, W. Hofmann \& F. M. Rieger (Melville, NY: AIP), 281

Araudo, A. T., Romero, G. E., Bosch-Ramon, V., \& Paredes, J. M. 2007, A\&A, 476, 1289

Aspin, C., \& Geballe, T. R. 2007, A\&A, 266, 219

Bell, A. R. 1978, MNRAS, 182, 147

Blondin, J. M., Konigl, A., \& Fryxell, B. A. 1989, ApJL, 337, L37

Bosch-Ramon, V., Romero, G. E., Araudo, A. T., \& Paredes, J. M. 2010, A\&A, 511, A8

Carrasco-González, C., Galván-Madrid, R., Anglada, G., et al. 2012, ApJL, 752, L29

Carrasco-González, C., Rodríguez, L. F., Anglada, G., et al. 2010, Sci, 330, 1209

Chevalier, R. A. 1999, ApJ, 511, 798

Curiel, S., Rodríguez, L. F., Moran, J. M., \& Canto, J. 1993, ApJ, 415, 191

Fernández-López, M., Curiel, S., Girart, J. M., et al. 2011a, AJ, 141, 72

Fernández-López, M., Girart, J. M., Curiel, S., et al. 2011b, AJ, 142, 97

Fernández-López, M., Girart, J. M., Curiel, S., et al. 2013, ApJ, 778, 72

Garay, G., Ramírez, S., Rodríguez, L. F., Curiel, S., \& Torrelles, J. M. 1996, ApJ, 459, 193

Girart, J., Estalella, R., \& Ho, P. T. P. 1998, ApJL, 495, L59

Girart, J. M., Rodríguez, L. F., Anglada, G., et al. 1994, ApJL, 435, L145

Gómez, Y., Rodríguez, L. F., Girart, J. M., Garay, G., \& Martí, J. 2003, ApJ, 597,414

Heathcote, S., Reipurth, B., \& Raga, A. C. 1998, AJ, 116, 1940

Martí, J., Rodríguez, L. F., \& Reipurth, B. 1993, ApJ, 416, 208

Martí, J., Rodríguez, L. F., \& Reipurth, B. 1995, ApJ, 449, 184

Martí, J., Rodríguez, L. F., \& Reipurth, B. 1998, ApJ, 502, 337

Masqué, J. M., Girart, J. M., Beltrán, M. T., Estalella, R., \& Viti, S. 2009, ApJ, 695,1505

Masqué, J. M., Girart, J. M., Estalella, R., Rodríguez, L. F., \& Beltrán, M. T. 2012, ApJL, 758, L10

Ostrowski, M. 1999, A\&A, 345, 256

Park, J., Caprioli, D., \& Spitkovsky, A. 2015, PhRvL, 114, 085003

Pravdo, S. H., Tsuboi, Y., \& Maeda, Y. 2004, ApJ, 605, 259

Raga, A. C., Noriega-Crespo, A., \& Velázquez, P. F. 2002, ApJL, 576, L149

Reipurth, B., \& Graham, J. A. 1988, A\&A, 202, 219

Rodríguez, L. F., Curiel, S., Moran, J. M., et al. 1989, ApJL, 346, L85

Rodríguez, L. F., Delgado-Arellano, V. G., Gómez, Y., et al. 2000, AJ, 119,882

Rodríguez, L. F., Garay, G., Brooks, K. J., \& Mardones, D. 2005, ApJ, 626,953

Rodríguez, L. F., Moran, J. M., Ho, P. T. P., \& Gottlieb, E. W. 1980, ApJ, 235,845

Wilner, D. J., Reid, M. J., \& Menten, K. M. 1999, ApJ, 513, 775 